\title{
PET/MR Imaging in the Detection and Characterization of Pulmonary Lesions: Technical and Diagnostic Evaluation in Comparison to PET/CT
}

\author{
Isabel Rauscher*1, Matthias Eiber*1, Sebastian Fürst ${ }^{2}$, Michael Souvatzoglou ${ }^{2}$, Stephan G. Nekolla ${ }^{2}$, Sibylle I. Ziegler ${ }^{2}$, \\ Ernst J. Rummeny ${ }^{1}$, Markus Schwaiger ${ }^{2}$, and Ambros J. Beer ${ }^{2}$ \\ ${ }^{I}$ Department of Diagnostic and Interventional Radiology, Technische Universität München, Munich, Germany; and ${ }^{2}$ Department of \\ Nuclear Medicine, Technische Universität München, Munich, Germany
}

Fully integrated PET/MR imaging holds great promise as a novel hybrid imaging modality in oncology and might offer advantages to PET/CT in many instances, especially because of the superior softtissue contrast of MR imaging, compared with CT. However, lung metastases are a frequent finding in oncologic patients, and for imaging of the lung CT is still the modality of choice. Thus, we prospectively evaluated differences in quality, detection rate, size, and radiotracer uptake of pulmonary lesions in ${ }^{18} \mathrm{~F}-\mathrm{FDG}$ PET/CT and PET/MR imaging. Methods: Institutional review board approval and informed consent were obtained. Forty patients (23 men, 17 women; mean age $\pm S D, 53.2 \pm 13.1$ y) underwent a single-injection dualimaging protocol with ${ }^{18} \mathrm{~F}-\mathrm{FDG}$ PET/CT and PET/MR imaging. Pulse sequences for the lung included T1-weighted VIBE (volumetric interpolated breath-hold examination) Dixon for attenuation correction and contrast-enhanced VIBE pulse sequences. All patients underwent a diagnostic CT of the chest in deep inspiration, which also served as a standard of reference. Two masked readers assessed in consensus all images randomly concerning quality, detection, standardized uptake value (SUV), and size of pulmonary nodules. Correlations were performed using linear correlation. Results: Overall, 47 pulmonary lesions (mean size $\pm S D, 10.0 \pm 11.4 \mathrm{~mm}$; range, $2-60 \mathrm{~mm}$ ) in 25 of 40 patients were detected. The PET datasets of PET/MR imaging and PET/CT revealed 22 of 47 pulmonary lesions with focal ${ }^{18} \mathrm{~F}-\mathrm{FDG}$ uptake. SUVs of lung lesions in PET/MR imaging and $\mathrm{PET} / \mathrm{CT}$ correlated significantly $(R=0.9 ; P=0.0001)$ and showed no significant difference (mean SUV PET/MR imaging, 6.3; PET/CT, 5.1; $P=0.388$ ). There was a significantly lower image quality comparing Dixon and VIBE sequence with CT whereas PET from PET/CT and PET from PET/MR imaging showed the same results (2.8). Dixon images detected 15 of 47 lung lesions whereas VIBE images detected 32 of 47 lesions, respectively. The detection rates for small lung lesions less than $1 \mathrm{~cm}$ in diameter $(n=33)$ of MR imaging was significantly lower, with a detection rate of 9 of 33 for the Dixon sequence and 15 of 33 for the VIBE sequence $(P<0.0001$ for VIBE and Dixon sequence). There was a high correlation of pulmonary lesion size of CT versus VIBE $(R=0.97)$. Conclusion: PET image quality and detection rate of ${ }^{18} \mathrm{~F}-\mathrm{FDG}$-positive lung lesions in $\mathrm{PET} / \mathrm{MR}$ imaging is equivalent to $\mathrm{PET} / \mathrm{CT}$ despite differences in attenuation-correction techniques. Additionally, a high linear correlation coefficient in the SUVs for the PET images from PET/CT and

Received Jul. 11, 2013; revision accepted Nov. 4, 2013.

For correspondence or reprints contact: Ambros Beer, Technische Universität München, Ismaninger Strasse 21, Munich, Germany 81675.

E-mail: ambros.beer@tum.de

${ }^{*}$ Contributed equally to this work.

Published online Mar. 20, 2014.

COPYRIGHT (C) 2014 by the Society of Nuclear Medicine and Molecular Imaging, Inc.
PET/MR imaging was found. The detection rate of lung lesions can be significantly improved by adding a diagnostic contrast-enhanced VIBE sequence to the PET/MR imaging protocol. However, the detection rate of small lung lesions is still inferior, compared with PET/CT with diagnostic CT of the chest.

Key Words: positron emission tomography; magnetic resonance imaging; computed tomography; hybrid imaging; pulmonary lesion

J Nucl Med 2014; 55:724-729

DOI: 10.2967/jnumed.113.129247

$\mathbf{P}$ ulmonary lesions are a common incidental finding in imaging studies although their evaluation is complex and still remains a diagnostic challenge. Advances in CT, MR imaging, and PET have improved their characterization, helping to differentiate benign and malignant lesions, which is important for the future prognosis of a patient (1). Several studies confirmed that ${ }^{18} \mathrm{~F}$-FDG PET is an accurate method for characterizing pulmonary lesions, with an overall sensitivity of $96.8 \%$ and a specificity of $77.8 \%$ (2). The development of integrated PET/CT as a combination of anatomic and metabolic imaging has also demonstrated an excellent performance in classifying pulmonary lesions (3). However, in PET/CT, pulmonary nodules smaller than $10 \mathrm{~mm}$ in diameter are often false-negative when looking at ${ }^{18} \mathrm{~F}$-FDG uptake only and in this case can be detected on the corresponding CT part only $(2,4)$. CT is still the method of choice for detecting small lung lesions, because MR imaging of the chest is limited by low proton densities of the lung parenchyma and fast transverse magnetization decay (5-7). With the development of PET/MR imaging whole-body staging examinations in oncology, the value of PET/MR imaging, compared with PET/CT, with respect to the detection of lung lesions is of specific interest. Recent studies in a small number of patients suggest the feasibility of whole-body PET/MR imaging in the assessment of lung cancer, with similar lesion-characterization and tumor-stage-comparing ability between PET/CT and PET/MR imaging (8). However, this study dealt with primary lung cancer staging and did not analyze the performance of PET/MR imaging for the evaluation of lung metastases, which might be substantially smaller. Moreover, the techniques for attenuation correction of PET data in PET/MR imaging are different from those in PET/CT. However, quantitative analysis of tracer uptake using the standardized uptake value (SUV) is essential for correct analysis of PET tracer distribution (9-13). Currently, in the commercially available whole-body fully integrated 
PET/MR imaging system (Biograph mMR; Siemens Medical Solutions) only a 2-point Dixon-based MR sequence is approved by the Food and Drug Administration for attenuation correction. First data using a whole-body fully integrated PET/MR imaging scanner indicate that the performance of PET in this system in general is equivalent to PET/CT (14). These initial results were not focused specifically on lung lesions, though. Moreover, the results of this former study were based on the comparison of low-dose CT and a low-resolution T1weighted Dixon VIBE (volumetric interpolated breath-hold examination) sequence used for attenuation correction. However, for CT imaging of the lungs, an examination of the chest in deep inspiration is state of the art $(15,16)$, whereas for MR imaging additional contrast-enhanced breath-hold sequences are mostly used (17).

Thus, the purpose of our study was 2-fold: first, to compare the performance of PET/MR imaging including diagnostic MR imaging sequences for detecting lung lesions, compared with PET/CT, in oncologic diseases; second, to analyze the significance of the different attenuation-correction techniques in PET/MR imaging, compared with PET/CT, for the evaluation of ${ }^{18} \mathrm{~F}-\mathrm{FDG}$-positive lung lesions.

\section{MATERIALS AND METHODS}

\section{Patient Population}

In this study, 40 randomly selected patients ( 23 men, 17 women; mean age $\pm \mathrm{SD}, 53.2 \pm 13.1 \mathrm{y}$; age range, 18-79 y), routinely referred to our institute for clinical ${ }^{18} \mathrm{~F}$-FDG PET imaging for staging or follow-up of malignant or suspected malignant disorders, were included. Patients with the following medical characteristics were recruited between July 2011 and August 2012: bronchial carcinoma, $n=2$; breast cancer, $n=4$; thyroid cancer, $n=5$; lymphoma, $n=3$; head and neck cancer, $n=20$; carcinoma of unknown primary, $n=1$; testicular cancer, $n=1$; adrenal gland cancer, $n=1$; primary liver tumor, $n=1$; rectal cancer, $n=1$; and liver hemangioendothelioma, $n=1$.

The local institutional review board approved this study. Written informed consent was obtained from all patients. Inclusion criteria were informed consent, ability to undergo another scan after the PET/CT examination, and clinical indication for an ${ }^{18} \mathrm{~F}-\mathrm{FDG}$ PET/CT scan. Exclusion criteria were pregnancy, age below $18 \mathrm{y}$, and contraindications for MR imaging examinations (e.g., magnetic metal implants, pacemakers).

\section{Imaging Protocol}

All subjects underwent a single-injection dual-imaging protocol. After completion of the PET/CT scan, patients were subsequently positioned on the PET/MR imaging scanner with the smallest possible temporal delay.

$\mathrm{PET} / \mathrm{CT}$ images were acquired according to standard clinical protocols, as previously reported (10) on a Biograph Sensation 64 PET/CT scanner (Siemens Medical Solutions) (18). Patients fasted for at least $6 \mathrm{~h}$ before scanning, and blood glucose levels were measured just before injection to ensure a value below $150 \mathrm{mg} / \mathrm{dL}$. Patients were injected with $383 \pm 35 \mathrm{MBq}$ of ${ }^{18} \mathrm{~F}-\mathrm{FDG}$ intravenously depending on their body weight, and the acquisition was started at $87 \pm 35 \mathrm{~min}$ after injection, moving from the pelvis to the head. Acquisition time was 2 or 3 min per bed position, with 5-6 bed positions (each $21 \mathrm{~cm}$ ) covering the trunk of the patients. For attenuation-correction purposes and anatomic correlation, a low-dose CT scan $(120 \mathrm{keV}, 20 \mathrm{mAs}$, no intravenous contrast) was acquired in shallow expiration. When clinically indicated, a diagnostic CT $(120 \mathrm{kV}, 240 \mathrm{mAs}$, $0.5 \mathrm{~s}$ per rotation, $5-\mathrm{mm}$ slice thickness, portal venous phase $80 \mathrm{~s}$ after the injection of $80-120 \mathrm{~mL}$ intravenous contrast agent [Imeron 300; Bracco Imaging Deutschland $\mathrm{GmbH}]$ ) was performed. In these cases, the diagnostic CT was used for attenuation correction. For evaluation of the lungs, a separate low-dose CT of the chest in deep inspiration was performed in all patients ( $25 \mathrm{mAs}, 120 \mathrm{kV}, 0.5 \mathrm{~s}$ per rotation, 5 -mm slice thickness). CT images of the chest were reconstructed with a standardized window setting for lung parenchyma visualization (window level, -450 Hounsfield units
[HU]; window width, 1,800 HU) using a sharp convolution kernel. Nonoverlapping images were reconstructed with a slice thickness of $5 \mathrm{~mm}$.

All PET/MR imaging examinations were performed using a fully integrated whole-body hybrid PET/MR imaging system (Biograph mMR; Siemens Healthcare). The technical specifications have been described previously (19-21).

On average, PET/MR imaging was started $125 \pm 25$ min after injection. The combined imaging protocol was as follows: after obtaining a localizer MR image to define the bed positions, combined PET/MR imaging was initiated with 3-5 bed position at 4-min acquisition times per bed position. First, a coronal 2-point Dixon 3-dimensional VIBE T1-weighted MR image sequence was acquired in each bed position in shallow expiration, which was used for the generation of attenuation maps (9). The technical parameters for this sequence are summarized in Table 1. To minimize artifacts from incomplete breath-hold, a centric k-space acquisition was chosen (22). Together with the start of this Dixon MR image sequence, the PET acquisition started simultaneously in the same bed position, thus ensuring optimal temporal and regional correspondence between MR imaging and PET data. In the thorax and abdomen region, the MR images were acquired during breath-hold in end-expiratory phase. An additional fat-suppressed contrast-enhanced 3-dimensional VIBE pulse sequence was performed over the lung in deep inspiration with the sequence parameters summarized in Table 1.

\section{Data Processing and Image Reconstruction}

Identical reconstruction and correction algorithms for PET data obtained on the PET/CT and on the PET/MR imaging scanner were used. For both modalities, emission data were corrected for randoms, dead time, scatter, and attenuation. A 3-dimensional ordered-subset expectation maximization iterative reconstruction algorithm was applied, with 3 iterations and 21 subsets, $4 \mathrm{~mm}$ in full width at half maximum gaussian smoothing, and a zoom of 1 . Attenuation maps were obtained from the CT data by bilinear transformation, as implemented in the postprocessing software of the PET/CT scanner and used for attenuation correction of the PET/CT data, as previously described (11).

For a subset of patients with ${ }^{18} \mathrm{~F}-\mathrm{FDG}$-positive lesions, the PET data from PET/MR imaging were reconstructed using the HUs from normal lung from the corresponding CT data of the patient instead of the fixed $\mu$ value of 224 normally used in PET/MR imaging for the lungs.

\section{MR Imaging-Based Attenuation Correction}

For attenuation correction of the PET data from the PET/MR imaging, attenuation maps were generated as recently published $(9,10)$. The lungs were identified by connected-component analysis of the air in the inner part of the body. By application of a morphologic closing filter, virtual air artifacts induced by the absence of an MR imaging signal in cortical bone, heart, and aorta (because of blood flow) were corrected (12).

\section{Image Analysis}

Images were analyzed by 2 readers ( 1 nuclear medicine physician and radiologist and 1 radiologist) masked to the patient history. The different datasets of each patient (CT, T1-weighted VIBE Dixon sequence, T1-weighted VIBE fat-saturated sequence, PET of PET/MR imaging, and PET of PET/CT) were evaluated with at least a 2 -wk interval. The entire rating procedure was performed on a dedicated workstation using software (Syngo MMWP [workstation] and Syngo TrueD [software]; Siemens Medical Solutions).

The ${ }^{18} \mathrm{~F}-\mathrm{FDG}$ PET data from both modalities and CT, Dixon, and VIBE sequences were rated for every patient with regard to overall image quality: 0 , non-diagnostic image quality; 1 , low image quality (distinct artifacts, strong image noise); 2 , satisfying image quality (little artifacts, moderate image noise); and 3, excellent image quality (no artifacts, low image noise).

Subsequently, in a first step, the detection rate of pulmonary lesions (benign or malignant) in the 2 different MR image sequences (Dixon and VIBE sequences) and both PET datasets (including both the non-attenuation-corrected and the attenuation-corrected PET) was 
TABLE 1

Technical Parameters of Different MR Imaging Sequences Used in This Study

\begin{tabular}{lcc}
\hline \multicolumn{1}{c}{ Sequence } & T1-weighted VIBE Dixon & T1-weighted VIBE fat saturated \\
\hline Repetition time/echo time $(\mathrm{ms})^{*}$ & $3.61 / 1.23$ and 2.46 & $3.29 / 1.16$ \\
Slice thickness $(\mathrm{mm})$ & 3.1 & 5 \\
Gap (\%) & 0 & 0 \\
Matrix & $79 \times 192$ & $320 \times 240$ \\
Field of view $(\mathrm{mm})$ & 500 & 460 \\
Bandwidth $(\mathrm{kHz})$ & 960 & 540 \\
Voxel size $\left(\mathrm{mm}^{3}\right)$ & $4.1 \times 2.6 \times 3.1$ & $1.8 \times 1.4 \times 5$ \\
Flip angle $\left(^{\circ}\right)$ & 10 & 9 \\
\% phase field of view & 65.5 & 75.0 \\
Acquisition time (min:sec) & $0: 19$ & $0: 16$ \\
No. of excitations & 1 & 1 \\
Integrated parallel acquisition technique factor & 2 & 2 \\
& & \\
\hline
\end{tabular}

assessed ( 0 , absence of a lung lesion; 1 , presence of one or several lung lesions), with CT being the standard of reference. If a lung lesion was found, its dignity was rated on a 3-point-scale (1, most likely unspecific/ benign; 2, indeterminate; and 3 suggestive of malignancy) in each morphologic and both PET datasets. In addition, in every morphologic dataset its size in millimeters was measured. For rating of the PET/CT data, PET images were screened for any suspected focal uptake. The standard of reference was based on the CT scan including both past and follow-up examinations as well as medical history and all other clinical information.

\section{Quantitative Assessment}

For quantitative comparison of the PET and CT data, an SUV analysis of tracer uptake in the suspected lesions and in the right and left normal lung was performed. Volumes of interest were placed over matching corresponding ${ }^{18} \mathrm{~F}-\mathrm{FDG}$ images of suspected lung lesions. To calculate SUVs, an isocontour volume of interest including all voxels above $50 \%$ of the maximum was created covering the whole lesion volume. Within all volumes of interest, mean SUV ( $\left.\mathrm{SUV}_{\text {mean }}\right)$ and maximum SUV $\left(\mathrm{SUV}_{\mathrm{max}}\right)$ were measured. For areas of normal lungs, regions of interest (3-cm diameter) were placed in the right and left lung, respectively, in areas without pathologic abnormalities. For CT scans, additional measurements of HUs of pulmonary lesions and HUs of normal right and left lung parenchyma were performed.

\section{Statistical Analysis}

Statistical analysis was performed using the MedCalc software package (version 7.2.0.2., for Windows [Microsoft]). Descriptive statistical evaluation was performed; differences in image quality and size of lung lesions were compared using a 2-sample Student $t$ test. Differences were deemed to be significant at a $P$ value of less than 0.05 . In addition, the mean $\mathrm{SUV}_{\text {mean }}$ and $\mathrm{SUV}_{\text {max }}$ of PET/CT and PET/MR imaging were correlated (correlation coefficient $R, P$ value, and 95\% confidence interval $[\mathrm{CI}]$ ), and a Bland-Altman plot was performed. To analyze the diagnostic performance of both modalities, the detection rate was calculated on the basis of the total lung lesions determined by the standard of reference.

\section{RESULTS}

\section{Detection of Benign and Malignant Pulmonary Lesions}

Forty-seven pulmonary nodules were present in 25 of 40 patients ( $n=14>1 \mathrm{~cm}, n=33<1 \mathrm{~cm}$ diameter) as seen in Table 2 . According to the standard of reference, 24 pulmonary lesions seen were categorized as malignant and 23 were categorized as benign. Dixon images detected 15 of 47 lung lesions (no false-positive findings), and VIBE images detected 32 of 47 lesions (3 lesions false-positive), respectively. The detection rates for small lung lesions less than $1 \mathrm{~cm}$ in diameter $(n=33$ in CT) on MR images were significantly lower, with a detection rate of 9 of 33 for the Dixon sequence and 15 of 33 for the VIBE sequence $(P<0.0001$ for VIBE and Dixon sequence). The characterization of all lung lesions in CT, VIBE, and Dixon is shown in Table 3. In CT, most of the lung lesions were categorized as most likely unspecific/benign or suggestive of malignancy, and only 2 of 47 lung lesions were classified as indeterminate. However, in the Dixon sequence $27 \%$ and in VIBE $22 \%$ of the lung lesions were classified as indeterminate.

\section{Image Quality in CT, MR Imaging, and PET and Assessment of Lesion Size}

The results of overall image quality (mean, SD, and $P$ value) in CT, MR imaging, and PET are seen in Table 4. As suspected, CT revealed the highest score, with an overall excellent image quality score of 3 in all datasets. There was a significantly lower image quality when comparing Dixon and VIBE sequences with CT whereas PET from PET/CT and PET from PET/MR imaging showed similar results.

There was no significant difference in size of pulmonary lesions between CT, compared with Dixon $(P=0.1)$, and VIBE sequence, compared with CT $(P=0.8)$. Furthermore, there was a strong correlation between the lesions size of CT and VIBE $(R=0.97 ; P<0.0001$; 95\% CI, 0.9307-0.9852) and between CT and Dixon sequence $(R=$ $0.55 ; P=0.0328$; 95\% CI, 0.0556-0.8297); detailed information with a graphical correlation analysis and a Bland-Altman plot can be seen in Supplemental Figure 1 (supplemental materials are available at http://jnm.snmjournals.org).

The $\mu$ map of the lung was identified correctly in 35 of 40 patients.

\section{Correlation Between SUVs from PET/CT and PET/MR Imaging}

The PET datasets of PET/MR imaging and PET/CT revealed 22 of 47 pulmonary lesions with focal ${ }^{18}$ F-FDG uptake in 10 of the 40 patients (21\% of all lung lesions). In PET/MR imaging, mean $\mathrm{SUV}_{\text {mean }}$ was $6.3 \pm 5.5$ and mean $\mathrm{SUV}_{\max }$ was $9.2 \pm 8.3$. Corresponding $\mathrm{SUV}_{\text {mean }}$ and $\mathrm{SUV}_{\text {max }}$ of PET/CT were $5.1 \pm 4.0$ and $7.3 \pm 5.8$, respectively. No statistical difference could be found between mean $\mathrm{SUV}_{\mathrm{CT}}$ and $\mathrm{SUV}_{\mathrm{MR}}$ (SUV of the PET of PET/CT and SUV of the PET of PET/MR imaging, respectively; $P=0.388$ ) as well as maximum $\operatorname{SUV}_{\mathrm{CT}}$ and $\operatorname{SUV}_{\mathrm{MR}}(P=0.362)$. Moreover, a high linear correlation coefficient in the $\mathrm{SUV}_{\text {mean }}$ and $\mathrm{SUV}_{\max }$ for the PET images from PET/CT and PET/MR imaging was found, as shown in 
Figure 1 for $\mathrm{SUV}_{\text {mean }}$ (correlation coefficient $R=0.90 ; P<0.0001$; 95\% CI, 0.77-0.96 for $\mathrm{SUV}_{\text {mean }}$, and $R=0.91 ; P<0.0001 ; 95 \% \mathrm{CI}$, $0.80-0.96$ for $\left.\mathrm{SUV}_{\max }\right)$. Correlation analysis and Bland-Altman plot for $\mathrm{SUV}_{\max }$ and normal lung tissue can be found in Supplemental Figure 2.

Because there was tendency for higher SUVs in PET/MR imaging, we reconstructed the PET data of PET/MR imaging also using the $\mathrm{HU}$ of the normal lungs as determined by the corresponding CT of the same patient and not the fixed $\mu$ value of 224 for lungs normally used in PET/MR imaging. This was done for 7 patients with 13 lesions. The mean values and SDs for $\mathrm{SUV}_{\text {mean }}$ were $3.65 \pm 2.92$. The corresponding values for PET/CT were $3.73 \pm 3.12$ and for PET/MR imaging with fixed $\mu$ value, $4.08 \pm 3.25$.

Figures 2 shows representative images (CT, VIBE, and Dixon images as well as PET of PET/CT) of a patient with a small lung lesion in the left upper lung lobe with no suspected ${ }^{18} \mathrm{~F}-\mathrm{FDG}$ uptake. The lung lesion was seen in the CT and VIBE sequence but not in the Dixon sequence. Additional representative images are shown in the supplemental material.

\section{DISCUSSION}

The results of this study indicate that there is no significant difference between PET datasets of the lung in PET/MR imaging and PET/CT concerning image quality and lesion characterization. Adding a diagnostic MR imaging sequence for lung analysis in deep inspiration significantly increases the pulmonary lesion detection rate in PET/MR imaging; however, PET/MR imaging is still inferior to PET/CT for diagnostic CT of the lung.

Schwenzer et al. were the first, to our knowledge, to compare the performance of whole-body hybrid PET/MR imaging and PET/CT in patients with lung lesions; however, this study dealt with primary staging of lung cancer with rather large lesion sizes (8). We focused on comparing the performance of PET/MR imaging and PET/CT for lung lesions in cancer patients in general, which is of importance because in this indication the lesions usually are substantially smaller and the requirements for imaging are different from those with primary staging of lung cancer. Most of the lung lesions in our patient population were smaller than $1 \mathrm{~cm}$ (33/47), which explains the lower detection rate in our study. For these small lesions, detection is especially difficult in MR imaging because of the low proton density of lung tissue and the limited spatial resolution (23). A study comparing low-dose CT in shallow breathing and Dixon-based MR imaging (used for attenuation correction) of ${ }^{18} \mathrm{~F}-\mathrm{FDG}$-positive and -negative pulmonary nodules in a trimodality PET/CT/MR setup showed similar detection rates and localization accuracy. In our study, the Dixon sequence for attenuation correction performed significantly worse and was inferior to both the $\mathrm{CT}$ and the dedicated contrast-enhanced T1-weighted VIBE sequence in inspiration $(17,24)$. These discrepancies might, on the one hand, be explained by methodologic differences, because different systems and sequences were used. Also, we used diagnostic CT of the chest in deep inspiration as a standard of reference and not a CT in shallow breathing. The detection of lung lesions in CT not performed in deep inspiration might be hampered, especially at the base of the lungs because of suboptimal expansion of lung tissue, which might contribute to the better performance of CT in our study $(16,25)$.

We also evaluated a dedicated contrast-enhanced T1-weighted VIBE sequence in inspiration for hybrid PET/MR imaging. When the detection rate of VIBE was compared with CT scan, 32 of 47 of the lung lesions were seen in VIBE as well; the ones that were not detected $(n=15)$ were mostly lung lesions with a diameter less than $1 \mathrm{~cm}$. Thus, for pulmonary diagnostic in MR imaging an additional imaging sequence (e.g., contrast-enhanced VIBE) is essential, although the 
TABLE 3

Characterization of Lung Lesions in CT, VIBE, and Dixon Sequences

\begin{tabular}{lccc}
\hline Modality & 1-most likely unspecific/benign & 2-indeterminate & 3-suggestive of malignancy \\
\hline CT $(n=47)$ & 21 & 2 & 24 \\
VIBE $(n=32)$ & 9 & 7 & 16 \\
Dixon $(n=15)$ & 2 & 4 & 9 \\
\hline
\end{tabular}

diagnostic value of contrast-enhanced VIBE is still inferior to CT, especially for small lung lesions. However, the significance of this finding might vary depending on the specific clinical scenario. For some patient groups, the detection rate of the contrast-enhanced VIBE sequence might be sufficient, for example, in prostate cancer patients with early biochemical relapse, because in this group lung metastases are rare and when present usually they are larger than $1 \mathrm{~cm} \mathrm{(26).} \mathrm{Also}$ many patients might already present with diagnostic CT images including the chest before undergoing the PET/MR examination. However, for most oncologic patients without prior CT of the chest, detection also of small lung nodules usually matters. Thus, we feel that a low-dose unenhanced CT of the lungs should be added to the PET/MR imaging in these cases, although future prospective studies finally have to define the value of PET/MR imaging, including dedicated sequences for lung imaging for specific oncologic indications.

In our study, we had 3 false-positive lung lesions in the VIBE sequence, compared with CT, meaning by our definition lesions seen in MR without any correlate in the respective CT scan. These false-positives were in 2 cases vascular structures in the lung periphery misinterpreted as nodules and in 1 case a lymph node in the paraaortal fat tissue of the thoracic aorta wrongly localized and thus misclassified as a lung nodule. In our experience the differential diagnosis of lesions seen in MR imaging was more difficult than in CT, as shown by the higher rate of indeterminate findings in MR imaging, compared with CT. In CT, characteristics such as the shape and density of a lesion and the imaging characteristics of the surrounding lung parenchyma might all help in differentiating malignant from benign lesions, for example, infectious infiltrates (27). As a consequence, some ${ }^{18} \mathrm{~F}-\mathrm{FDG}$-avid lesions with a correlate in MR imaging were wrongly classified as probably malignant in PET/MR imaging but were correctly classified in PET/CT by the CT information as probably infectious (benign), which was proven by follow-up imaging.

TABLE 4

Image Quality of CT, MR Imaging, and PET

\begin{tabular}{lll}
\hline \multicolumn{1}{c}{ Dataset } & \multicolumn{1}{c}{ Sequence } & Mean \pm SD \\
\hline Morphologic & VIBE & $2.60 \pm 0.63^{\dagger \S}$ \\
& CT & $3.00 \pm 0^{\dagger \ddagger}$ \\
& Dixon & $2.70 \pm 0.46^{\ddagger \S}$ \\
PET & PET AC-CT & $2.80 \pm 0.41^{\| \prime}$ \\
& PET AC-MR imaging & $2.83 \pm 0.38^{\| \prime}$ \\
& PET NAC-CT & $2.98 \pm 0.16^{\natural}$ \\
& PET NAC-MR imaging & $2.90 \pm 0.30^{\natural}$ \\
\hline
\end{tabular}

\footnotetext{
${ }^{\dagger} P=0.0003^{\star}$.

${ }^{\ddagger} P=0.0002^{*}$.

${ }^{\S} P=0.33$

$\| P=0.74$

" $P=0.18$

*Indicates a significant difference between two sequences.

$\mathrm{AC}=$ attenuation correction; $\mathrm{NAC}=$ non-attenuation correction.
}

Concerning the quality of the PET component of PET/MR imaging versus PET/CT for lung lesions, we found a comparable image quality and detection rate for both systems in the qualitative comparison, with highly correlating SUVs in the quantitative analysis. This finding suggests that PET/MR imaging is robust for the assessment of lung lesions and comparable to PET/CT despite differences in detector technology and attenuation correction. It has to be stressed, however, that both scanners at the time of the study did not use time-of-flight (TOF) technology. It is known that TOF increases the performance of PET, especially for small structures (28). Thus, the overall performance for ${ }^{18} \mathrm{~F}-\mathrm{FDG}$ PET is expected to improve also for detection of small lung lesions using TOF technology, which is, however, not available in the current PET/MR imaging system used here.

Contrary to previous studies focusing on lesions outside the lungs, we could see a tendency for slightly higher SUVs in PET/MR imaging, compared with PET/CT, which was not statistically significant (14). One potential explanation might be that the $\mu$ values from PET/CT for the lung were lower in the patients with lesions than the fixed value of 224 used in PET/MR imaging for lung tissue. We thus recalculated the $\mu$ values for lung in PET/CT for a subset of patients with lung lesions based on the HUs measured in normal lung in these patients and reconstructed the PET data of PET/MR imaging of the patients with ${ }^{18} \mathrm{~F}-\mathrm{FDG}$-avid lesions using

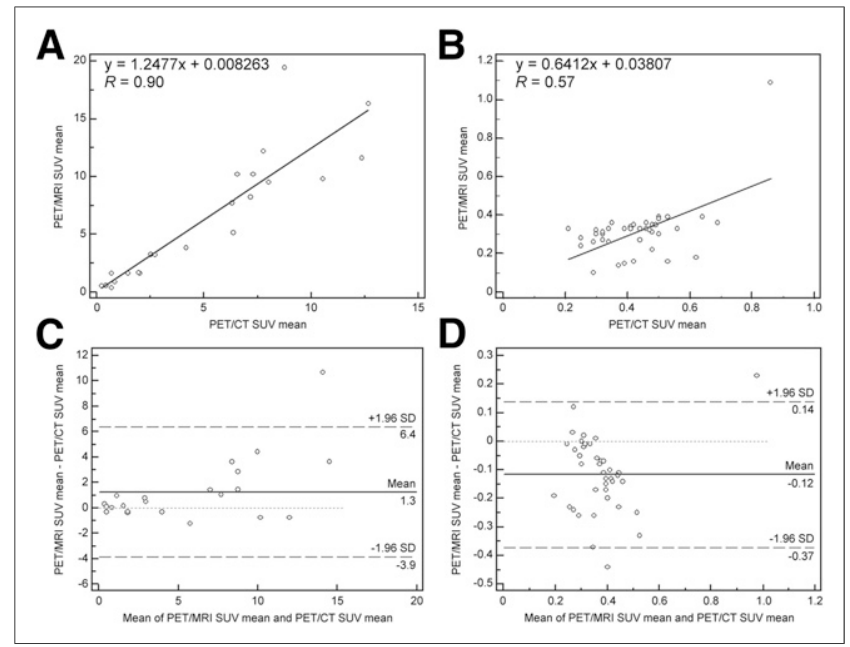

FIGURE 1. Correlation analysis of tracer uptake between PET/CT and subsequent PET/MR imaging as assessed by SUV mean in lung lesions (A) and normal lung parenchyma (B). $x$-axis displays quantitative values as obtained by PET/CT, and $y$-axis displays corresponding values by PET/ MR imaging. High correlations as expressed by Spearman correlation coefficient $(R)$ are found for $\mathrm{SUV}_{\text {mean }}(0.9$ for normal lung parenchyma, 0.57 for lung lesions) between findings from both modalities. Difference between 2 SUV measurements is shown by Bland-Altman (for SUV mean $_{\text {in normal lung }}$ parenchyma [C] and for $S V_{\text {mean }}$ in lung lesions [D]) on which difference between 2 SUV measurements is plotted against their average. For

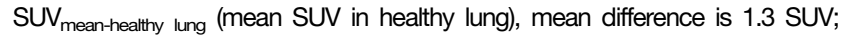

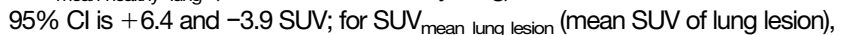
mean difference is -0.12 SUV; $95 \% \mathrm{Cl}$ for $S \mathrm{SU}_{\max }$ is +0.14 and $-0.37 \mathrm{SUV}$. 


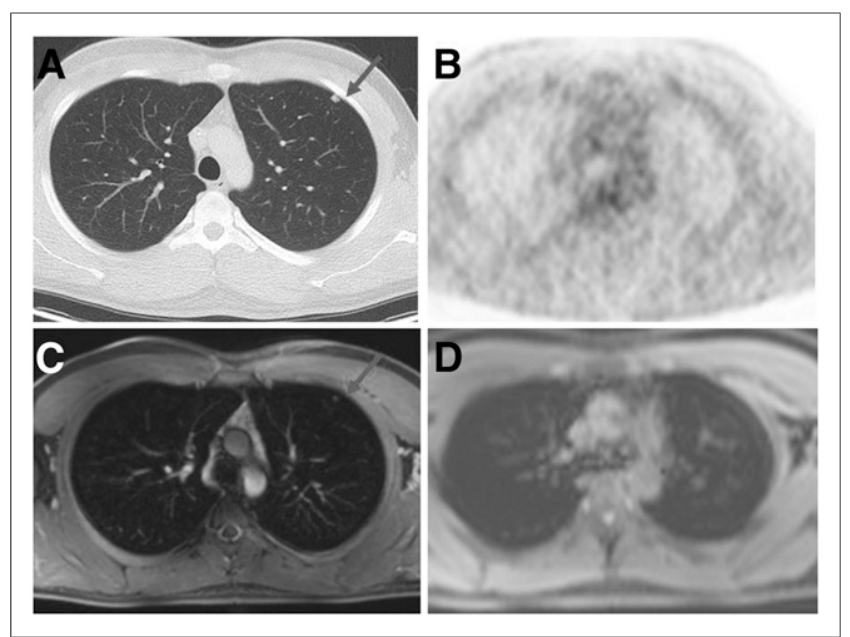

FIGURE 2. A 26-y-old patient with testicular cancer. (A, C, and D) Morphologic datasets (axial CT [A], axial VIBE [C], and axial Dixon sequence [D] [in phase]). (B) Corresponding PET of PET/CT. Lung lesion (arrow) was seen in CT and VIBE scan but not in Dixon sequence. Additionally, there was no suspected ${ }^{18} \mathrm{~F}$-FDG uptake.

the respective $\mu$ values from CT. This resulted in lower SUVs in the newly reconstructed PET images from PET/MR imaging, which now were slightly lower than the PET/CT data, suggesting a tendency for overestimation of the true $\mu$ values by the fixed value used in PET/MR imaging in our patient population. However, other factors might also play a role, such as surface coils or different scanner/ detector geometry. Also, biologic factors might contribute, such as a more pronounced trapping of tracer in malignant lesions due to the delayed imaging time point of PET/MR imaging; however, as mentioned before, the latter could not be observed for lesions outside the lung up to now in other comparative studies of PET/CT and PET/MR imaging with single-injection dual-imaging protocols.

Our study had some limitations. For ethical reasons, no histopathology as the gold standard for pulmonary lesions could be provided. However, in our study we were mainly interested in the general performance of PET/MR imaging versus PET/CT in the detection of lung lesions and did not focus primarily on the classification of the lesions. Moreover, because of the time-consuming PET/CT and PET/MR imaging examination, the protocol in our study comprised only one additional diagnostic sequence for lung imaging (VIBE). It is known that the detection rate of MR imaging for small lung lesions can be improved using a multisequence protocol (17). Thus, potential improvements can be expected by multisequence protocols also including respiratory triggering and gating of PET and MR data.

\section{CONCLUSION}

Our study shows that ${ }^{18} \mathrm{~F}-\mathrm{FDG}$ PET performance in PET/MR imaging for the assessment of lung lesions is robust and equivalent to PET/CT despite differences in detector technology and attenuationcorrection techniques. The detection rate of lung lesions can be significantly improved by adding a diagnostic contrast-enhanced VIBE sequence in inspiration to the PET/MR imaging protocol. However, the detection rate of small lung lesions is still inferior, compared with PET/CT with diagnostic CT of the chest.

\section{DISCLOSURE}

The costs of publication of this article were defrayed in part by the payment of page charges. Therefore, and solely to indicate this fact, this article is hereby marked "advertisement" in accordance with 18 USC section 1734 . No potential conflict of interest relevant to this article was reported.

\section{REFERENCES}

1. Jadvar H, Alavi A, Gambhir SS. ${ }^{18}$ F-FDG uptake in lung, breast, and colon cancers: molecular biology correlates and disease characterization. J Nucl Med. 2009;50:1820-1827.

2. Gould MK, Maclean CC, Kuschner WG, Rydzak CE, Owens DK. Accuracy of positron emission tomography for diagnosis of pulmonary nodules and mass lesions: a meta-analysis. JAMA. 2001;285:914-924.

3. Kim SK, Allen-Auerbach M, Goldin J, et al. Accuracy of PET/CT in characterization of solitary pulmonary lesions. J Nucl Med. 2007;48:214-220.

4. Nomori H, Watanabe K, Ohtsuka T, et al. Evaluation of F-18 fluorodeoxyglucose (FDG) PET scanning for pulmonary nodules less than $3 \mathrm{~cm}$ in diameter, with special reference to the CT images. Lung Cancer. 2004;45:19-27.

5. Regier M, Schwarz D, Henes FO, et al. Diffusion-weighted MR-imaging for the detection of pulmonary nodules at 1.5 Tesla: intraindividual comparison with multidetector computed tomography. J Med Imaging Radiat Oncol. 2011;55:266-274.

6. Schroeder T, Ruehm SG, Debatin JF, et al. Detection of pulmonary nodules using a 2D HASTE MR sequence: comparison with MDCT. AJR. 2005;185:979-984.

7. Koyama H, Ohno Y, Kono A, et al. Quantitative and qualitative assessment of noncontrast-enhanced pulmonary MR imaging for management of pulmonary nodules in 161 subjects. Eur Radiol. 2008;18:2120-2131.

8. Schwenzer NF, Schraml C, Muller M, et al. Pulmonary lesion assessment: comparison of whole-body hybrid MR/PET and PET/CT imaging-pilot study. Radiology. 2012;264:551-558.

9. Martinez-Möller A, Souvatzoglou M, Delso G, et al. Tissue classification as a potential approach for attenuation correction in whole-body PET/MRI: evaluation with PET/CT data. J Nucl Med. 2009;50:520-526.

10. Eiber M, Martinez-Moller A, Souvatzoglou M, et al. Value of a Dixon-based MR/ PET attenuation correction sequence for the localization and evaluation of PETpositive lesions. Eur J Nucl Med Mol Imaging. 2011;38:1691-1701.

11. Kinahan PE, Hasegawa BH, Beyer T. X-ray-based attenuation correction for positron emission tomography/computed tomography scanners. Semin Nucl Med. 2003;33:166-179.

12. Delso G, Martinez-Moller A, Bundschuh RA, et al. Evaluation of the attenuation properties of MR equipment for its use in a whole-body PET/MR scanner. Phys Med Biol. 2010;55:4361-4374.

13. Hofmann M, Steinke F, Scheel V, et al. MRI-based attenuation correction for PET/MRI: a novel approach combining pattern recognition and atlas registration. J Nucl Med. 2008;49:1875-1883.

14. Drzezga A, Souvatzoglou M, Eiber M, et al. First clinical experience with integrated whole-body PET/MR: comparison to PET/CT in patients with oncologic diagnoses. J Nucl Med. 2012;53:845-855.

15. Allen-Auerbach M, Yeom K, Park J, et al. Standard PET/CT of the chest during shallow breathing is inadequate for comprehensive staging of lung cancer. $\mathrm{J} \mathrm{Nucl}$ Med. 2006;47:298-301.

16. Juergens KU, Weckesser M, Stegger L, et al. Tumor staging using whole-body highresolution 16-channel PET-CT: does additional low-dose chest CT in inspiration improve the detection of solitary pulmonary nodules? Eur Radiol. 2006;16:1131-1137.

17. Heye T, Ley S, Heussel CP, et al. Detection and size of pulmonary lesions: how accurate is MRI? A prospective comparison of CT and MRI. Acta Radiol. 2012;53:153-160.

18. Jakoby BW, Bercier Y, Watson CC, et al. Performance characteristics of a new LSO PET/CT scanner with extended axial field-of-view and PSF reconstruction. IEEE Trans Nucl Sci. 2009;56:633-639.

19. Delso G, Fürst S, Jakoby B, et al. Performance measurements of the Siemens mMR integrated whole-body PET/MR scanner. J Nucl Med. 2011;52:1914-1922.

20. Sommer G, Schaefer AO, Baumann T, et al. Sliding multislice MRI for abdominal staging of patients with pelvic malignancies: a pilot study. J Magn Reson Imaging. 2008;27:666-672.

21. Pichler BJ, Judenhofer MS, Catana C, et al. Performance test of an LSO-APD detector in a 7-T MRI scanner for simultaneous PET/MRI. J Nucl Med. 2006;47:639-647.

22. Maki JH, Chenevert TL, Prince MR. The effects of incomplete breath-holding on 3D MR image quality. J Magn Reson Imaging. 1997;7:1132-1139.

23. Biederer J, Schoene A, Freitag S, et al. Simulated pulmonary nodules implanted in a dedicated porcine chest phantom: sensitivity of MR imaging for detection. Radiology. 2003;227:475-483.

24. Puderbach M, Hintze C, Ley S, et al. MR imaging of the chest: a practical approach at 1.5T. Eur J Radiol. 2007;64:345-355.

25. Bankier AA, O'Donnell CR, Boiselle PM. Quality initiatives. Respiratory instructions for CT examinations of the lungs: a hands-on guide. Radiographics. 2008;28:919-931.

26. Wallis CJ, English JC, Goldenberg SL. The role of resection of pulmonary metastases from prostate cancer: a case report and literature review. Can Urol Assoc J. 2011;5:E104-E108.

27. Tan BB, Flaherty KR, Kazerooni EA, et al. The solitary pulmonary nodule. Chest. 2003; 123:89S-96S.

28. Jakoby BW, Bercier Y, Conti M, Casey ME, Bendriem B, Townsend DW. Physical and clinical performance of the mCT time-of-flight PET/CT scanner. Phys Med Biol. 2011;56:2375-2389. 